\title{
„Der Neurologe hätte die Nervenleitgeschwindigkeit messen müs- sen.“- Das kommunikative Aushandeln des Expertenstatus in deutschsprachigen Diskussionsforen der Medizin.
}

\author{
Nadine Rentel (Zwickau)
}

\begin{abstract}
In online communication, the social identity of the persons involved is negotiated throughout communication processes with other members of a discourse community. In discussion fora in the domain of medicine, the focus lays on the discussion of the expert status of the users. This contribution aims at describing linguistic strategies that are used in order to create a positive self-image as medical expert in a community of practice. The study is based on a corpus of three selected German discussion fora concerning medical issues. Besides the need to boost the own expert status, we find examples in which users try to attenuate their knowledge claim. The results of the analysis indicate that for the domain of medicine, online and offline reality seem to be closely interwoven.
\end{abstract}

\section{$1 \quad$ Einleitung}

Seit ungefähr einem Jahrzehnt lässt sich ein gesteigertes Interesse und Bewusstsein für die eigene Gesundheit in der Bevölkerung konstatieren, welches sich dahingehend manifestiert, dass ein hoher Anteil von Menschen mit Zugang zum Internet gelegentlich oder regelmäßig Gesundheitsinformationen im Internet nutzt (cf. Kristiansen/Bonfadelli 2013: 240-243). Damit verbunden ist, zumindest in Deutschland, ein Trend zur Selbstmedikation: Anstatt bei akuten Beschwerden einen Arzt aufzusuchen, informieren sich die Betroffenen häufig erstmals auf einschlägigen Webseitenangeboten. Neben selbst Betroffenen nutzen auch Menschen, die einen Nahestehenden betreuen oder pflegen, das Informationsangebot im Internet (zu einer detaillierten Diskussion der Motive für gesundheitsbezogene Internetrecherchen siehe die Ausführungen in Kapitel 2). Als Folge dieses gestiegenen Informationsbedarfs zu medizinischen Themen haben die „Angebote zur Gesundheit auf öffentlichen Gesundheitsportalen, auf kommerziellen Websites oder in Gesundheitsforen, aber auch die Nutzungsmotivation und die Nutzung von gesundheitsrelevanter Information [...] in den letzten zehn Jahren stark zugenommen“ (Kristiansen/Bonfadelli 2013: 237). Nach der BITKOM-Studie aus dem Jahr 2011 lässt sich die Zahl für Deutschland auf etwa 28 Millionen Deutsche beziffern, 
wobei der typische Nutzer ${ }^{1}$ von Online-Angeboten jung (18-39 Jahre alt) und gut ausgebildet ist und über einen gehobenen sozialen Status verfügt (cf. Kristiansen/Bonfadelli 2013: 241).

Trotz des gesteigerten Interesses der Bevölkerung an Online-Gesundheitsangeboten ist für den Bereich der sprachwissenschaftlichen Forschung ein erhebliches Desiderat zu konstatieren. Nicht nur sind bereits durchgeführte Studien zu unterschiedlichen Textsorten weniger in der Sprachwissenschaft als in anderen Disziplinen (z. B. in der Kommunikationswissenschaft oder im Rahmen gesundheitssoziologischer Forschungen; cf. Schröder i. Ersch.) zu verorten, sondern für das Deutsche (und auch für die romanischen Sprachen) handelt es sich um ein erst seit einem knappen Jahrzehnt bearbeitetes Forschungsfeld und damit auf einige Fragestellungen bezogen um Neuland.

Um die oben skizzierte Forschungslücke zu schließen, werden im vorliegenden Beitrag am Beispiel einer qualitativen Analyse dreier ausgewählter deutschsprachiger Online-Foren aus dem Bereich der Medizin (www.gesundheit.de, www.gesundheit.com, www.onmeda.de), die auf Gesundheitsportalen verankert sind, sprachliche Strategien aufgezeigt, mit deren Hilfe sich Mitglieder der community of practice als Experten profilieren. Nach kurzen Überlegungen zur Gesundheitskommunikation im Internet und dem Versuch einer Funktionsbestimmung des ausgewählten Forentyps folgen einige Ausführungen zur Identitätskonstruktion in Sozialen Medien; damit eng verbunden ist die Diskussion der Konzepte „Experte " und "Laie“, die ebenfalls überblicksartig thematisiert wird. Im Anschluss werden die herausgearbeiteten Strategien dargestellt und anhand ausgewählter Korpusbelege illustriert.

\section{Gesundheitskommunikation im Internet}

Der Begriffsdiskussion im Kontext der Gesundheitskommunikation liegt entweder eine sehr breite (bezogen auf jedwede Kommunikation, deren Inhalte auf Gesundheit fokussiert sind) oder eine enger gefasste Definition zugrunde (wenn diese handlungsorientiert ausgerichtet ist in dem Versuch, das Verhalten von Individuen oder sozialen Systemen durch kommunikative Prozesse dahingehend zu beeinflussen, dass die Gesundheit gefördert wird). Der Begriff „Gesundheitskommunikation“ ist eine Übertragung aus dem Englischen (health communication). Health communication ist seit ca. 30 Jahren im angloamerikanischen Sprachraum eine fest etablierte Disziplin als Teilgebiet der Gesundheitswissenschaften - ganz anders als in Deutschland, wo Gesundheitskommunikation als Forschungsfeld innerhalb der deutschsprachigen Medienlinguistik erst seit ca. 10 Jahren im Fokus des Interesses steht (cf. Hurrelmann/Leppin 2001: 9). Hurrelmann/Leppin verstehen Gesundheitskommunikation als

die Vermittlung und den Austausch von Wissen, Meinungen und Gefühlen zwischen Menschen, die als professionelle Dienstleister oder Patienten/Klienten in den gesundheitlichen Versorgungsprozess einbezogen sind und/oder als Bürgerinnen und Bürger an Fragen von Gesundheit, Krankheit und öffentlicher Gesundheitspolitik interessiert sind.

(2001: 11)

Kreps/Bunaguro/Query (1998: 11) heben hervor, dass im Rahmen der Gesundheitskommunikation unterschiedliche Vermittlungskanäle relevant sind, die unterschiedliche Formen der

\footnotetext{
${ }^{1}$ Die maskulinen Personenbezeichnungen beziehen sich in diesem Beitrag gleichermaßen auf männliche wie auf weibliche Personen; in den Fällen, in denen sich das weibliche Geschlecht einer Kommunikationspartnerin eindeutig aus dem Kontext der diskutierten Beispiele ergibt, wird die feminine Form verwendet.
} 
Präsentation von Inhalten ermöglichen (zum Beispiel die direkte personale Gesundheitskommunikation im Gegensatz zur massenmedialen Vermittlung). Nach Hurrelmann/Leppin zeichnet sich Gesundheitskommunikation, bezogen auf die kommunikativen Parameter, durch folgende Charakteristika aus. Sie

kann direkt-personal, wie in der Arzt-Patient-Interaktion oder in einer Interaktion unter Experten erfolgen, oder [...] durch Medien vermittelt sein. Der Prozess der Kommunikation kann dabei mehr oder weniger symmetrisch verlaufen, das heißt eher den Charakter einer Information von Sender zu Empfänger haben, wie zum Beispiel (noch) beim Medium Fernsehen oder bei Plakatkampagnen, oder genuinen Austausch und gemeinsame Sinnkonstruktion repräsentieren, wie dies idealerweise in einem anamnestischen oder therapeutischen Arzt-Patient-Gespräch der Fall ist.

(2001: 11)

Baumann/Hurrelmann (2014: 13) unterscheiden vier Ebenen der Gesundheitskommunikation: Während sich die intrapersonale Kommunikation auf kommunikative Prozesse innerhalb einer Person bezieht (etwa die Überlegung, einen Arzt zu konsultieren oder nicht), umfasst die interpersonale Kommunikation kommunikative Prozesse zwischen mindestens zwei Kommunikationspartnern, zum Beispiel zwischen Arzt und Patient. Die Organisationskommunikation wiederum betrifft den Bereich der Kommunikation von und in Gesundheitsinstitutionen (zum Beispiel PR und Werbung von Krankenhäusern). Die im Rahmen des vorliegenden Beitrags analysierten Diskussionsforen lassen sich im Rahmen der vierten Kategorie, nämlich der massenmedialen bzw. gesellschaftlichen Kommunikationsprozesse, verorten, deren primäres Ziel die mediale Verbreitung von Gesundheitsinformationen ist.

Im Kontext der wissenschaftlichen Beschäftigung mit Diskussionsforen aus dem Bereich der Medizin stellt sich die grundlegende Frage nach den Motiven für die Informationsrecherche im virtuellen Raum. Die Auswertung des Materials legt nahe, dass neben der reinen Informationsbeschaffung der kommunikative Austausch mit anderen Betroffenen eine große Rolle zu spielen scheint (siehe dazu die Überlegungen $\mathrm{zu}$ den kommunikativen Funktionen in Kapitel 3). Kaltenborn (2001: 50-51) systematisiert die unterschiedlichen Nutzermotive folgendermaßen:

- Recherche nach Information und Wissen für förderliches Gesundheitsverhalten sowie für krankheitsadäquates Selbstmanagement

- Recherche nach Information und Wissen zur eigenen oder kooperativen Entscheidungsfindung mit dem Arzt

- Recherche nach Information zur Bewältigung der Vertrauens-/Misstrauensproblematik gegenüber dem behandelnden Arzt sowie gegenüber medizinischen Wissensangeboten; Ziel: Zusatzinformationen erhalten, die es den Patienten erlauben, die von ihrem Arzt empfohlenen Handlungsanweisungen beurteilen zu können; kritische Überprüfung von Diagnosen und Therapien

- Kommunikation und Austausch: Neben der Informationsbeschaffung steht für die User der social support im Fokus: Austausch eigener persönlicher Erfahrungen, Spenden von Trost, Diskussion alltäglicher Herausforderungen im Zusammenhang mit der Krankheit, etc.

Alle von Kaltenborn angeführten Nutzermotive lassen sich auch in den untersuchten Diskussionsforen nachweisen; diese manifestieren sich entweder konkret an der Textoberfläche, 
wenn die diskursive Konstruktion des Expertenstatus im Vordergrund steht, oder sind als ergänzende sprachliche Handlungen einzustufen (z. B. Spenden von Trost und Zuspruch, nachdem auf die eigene Erfahrung mit einer Krankheit verwiesen wurde).

\section{Die kommunikativen Rahmenbedingungen und Funktionen der Forenkommuni- kation}

Hypertexte sind komplexe textuelle Phänomene, denen oftmals nicht eine einzige kommunikative Funktion zugewiesen werden kann. Vielmehr handelt es sich um ein Bündel kommunikativer Funktionen (cf. Rehm 2005: 275). Diese Herausforderung der Funktionsdetermination ergibt sich vor allem aus der weiteren Verlinkung der Texte und der daraus resultierenden Verzeichnistiefe selbst innerhalb einzelner Themenbereiche. In medizinischen Diskussionsforen überlagern sich in der Realität ebenfalls zumeist mehrere kommunikative Funktionen im Rahmen eines Diskussionsbeitrags, jedoch scheint allgemein die Informationsfunktion zu dominieren, wenn Fachwissen über bestimmte Krankheitsbilder ausgetauscht wird; teilweise sind die Diskussionsbeiträge auch persuasiv oder instruierend intendiert, wenn Kommunikationspartner dazu aufgefordert werden, außerhalb der virtuellen Realität fachlich versierten medizinischen Rat einzuholen. Eine zentrale Rolle kommt weiterhin der Funktion der Selbstdarstellung sowie der Entlastungskommunikation (das „Teilen“ von Krankheitserfahrungen mit den Kommunikationspartnern) zu. ${ }^{2}$ Forenkommunikation dient des Weiteren dazu, soziale Kohäsion unter den Kommunikationspartnern zu schaffen (cf. Zappavigna 2011: 1); dies zeigt sich in den untersuchten Diskussionsforen, wenn User die Diskussionsbeiträge anderer Kommunikationspartner positiv bewerten und damit einen Beitrag zum in der virtuellen Kommunikation so wichtigen relational work leisten.

Die Kommunikationsteilnehmer in den untersuchten Foren sind einerseits interessierte Laien, die zu bestimmten medizinischen Fragen Rat suchen, andererseits handelt es sich um Personen, die sich als kompetent erachten, aufgrund eigener Erfahrungen oder einer gewissen Vorbildung solche Fragen zu beantworten und Ratschläge zu erteilen. Wie für die computervermittelte Kommunikation generell gilt auch für die analysierten Foren, dass die Sender zwar ein intendiertes Publikum mitdenken und sowohl den Inhalt als auch die Form ihrer Äußerungen daraufhin ausrichten, jedoch können sie nicht sicher sein, wer den Forenbeitrag rezipiert, da es sich in der öffentlichen Forenkommunikation um ein disperses Publikum handelt. ${ }^{3}$ Selbst bei der Annahme, dass es sich um einen relativ homogenen, thematisch einschlägig interessierten Adressatenkreis handelt, kann der (medizinische) Expertenstatus der Kommunikationsteilnehmer (und zwar sowohl auf der Sender- als auch auf der Empfängerseite) variieren. Daraus ergibt sich die Herausforderung des ,audience design“ (zum Terminus cf. Kunkel 2014: 409), d. h. das Anpassen von Inhalt und sprachlicher Form der Diskussionsbeiträge dahingehend, dass einerseits die Verständlichkeit (durch Berücksichtigen des vorausgesetzten Wissensstands der Rezipienten) sichergestellt ist, andererseits aber Autorität durch einen hohen Grad an sprachlich vermittelter Kompetenz geschaffen wird.

\footnotetext{
2 Zum Konzept des Sharing in der virtuellen Kommunikation (cf. Tienken 2013: 19).

${ }^{3}$ Marwick/Boyd (2010: 1) bezeichnen diesen Sachverhalt mit dem Terminus der „multiple audience“.
}

ISSN 1615-3014 
Die Kommunikationssituation in Foren ${ }^{4}$ ist als asynchron (raum-zeitlich entkoppelt), virtuell, indirekt und dialogisch zu charakterisieren. Das Kriterium der Dialogizität kann kontrovers diskutiert werden, denn nicht auf alle Forenbeiträge muss eine Reaktion seitens der discourse community erfolgen, obwohl dies von den Sendern in der Regel erwartet wird. 5 Zudem erscheint, anders als in der face-to-face-Kommunikation, die Antwort auf einen Forenbeitrag nicht unbedingt unmittelbar nach dessen Einstellen in das Forum. Auch kann die Zahl der einzelnen Dialogbeiträge, abhängig von der Relevanz eines Beitrags, variieren. Die mediale Realisierung ist schriftlich, wobei sich Einflüsse der gesprochenen Sprache in den Texten manifestieren, wie die Beispiele im Folgenden zeigen werden. ${ }^{6}$ Das Auftreten orthographischer und grammatischer Fehler ist umso auffälliger, da sich die Kommunikationspartner als Experten profilieren möchten; vermutlich ist die sprachliche Fehlerhaftigkeit den kommunikativen Parametern der Forenkommunikation geschuldet.

In der Online-Kommunikation spielt, wie bereits mehrfach erwähnt, das Identitätsmanagement eine zentrale Rolle. Auf das untersuchte Textkorpus bezogen, bezieht sich der Prozess der Identitätskonstruktion auf die Selbstdarstellung als medizinischer Experte. Anders als in der face-to-face-Kommunikation wird die soziale Identität der kommunizierenden Personen im kommunikativen Austausch mit den Mitgliedern der discourse community entworfen und bestätigt: „In other words, self-presentation is collaborative. Individuals work together to uphold preferred self-images of themselves and their conversation partners, through strategies like maintaining (or saving) face, collectively encouraging social norms, or negotiating power differentials and disagreements“ (Marwick/Boyd 2010: 10).

Dieser Prozess des identity management unterliegt dabei einer ständigen und dynamischen diskursiven (Re-)Validierung und ggf. Modifizierung, da Identität nicht als gegeben angesehen werden kann, sondern erst durch das Anwenden kommunikativer Praktiken entsteht. ${ }^{7}$ Aufgrund der spezifischen kommunikativen Parameter der computervermittelten Kommunikation wie beispielsweise der Existenz eines dispersen Publikums, des Fehlens einer gemeinsamen Wissensbasis der Kommunizierenden und der Restriktion non-verbaler Ressourcen, mittels derer der Expertenstatus in der Offline-Kommunikation generiert wird, haben verbale Strategien der positiven Selbstdarstellung ${ }^{8}$, die sich an der Textoberfläche der Diskussionsbeiträge manifestieren, einen zentralen Stellenwert inne. Eng mit dem Identitätsmanagement verbunden ist das Konzept des „,shared face“. Tajfel definiert „,social identity“ als „that part of an individual's self-concept which derives from his knowledge of his membership of a social group (or groups) together with the emotional significance attached to that membership“ (Tajfel 1974: 69). Ein Individuum ist also nicht nur bestrebt, sein eigenes Gesicht zu wahren, sondern auch das der übrigen Mitglieder der sozialen Gruppe, der es angehört. Wird ein Mitglied der community beleidigt, bedroht oder anderweitig herabgesetzt, empfindet das Indivi-

\footnotetext{
${ }^{4} \mathrm{Zu}$ einer detaillierten Diskussion der kommunikativen Parameter der Forenkommunikation (cf. Ehrhardt 2011; 2014).

${ }^{5}$ Nach Thaler (2012: 100) ist eine situationsadäquate sprachliche oder nicht-sprachliche Reaktion in der computervermittelten Kommunikation von grundlegender Bedeutung.

${ }^{6}$ Für eine vertiefte Diskussion der kommunikativen Parameter von Hypertexten (cf. Schröder 2013: 110-112).

${ }^{7}$ Zum Zusammenhang von face und Identität sowie zur Konstruktion und Verhandlung von Identität(en) im Rahmen der Interaktion zwischen Individuen (cf. Warm 2015: 288-289).

${ }^{8}$ In der einschlägigen Literatur werden diese mit den Termini „personal branding“ oder „,self-commodification“ bezeichnet (cf. Marwick/Boyd 2010).
} 
duum den Gesichtsverlust mit, da es Teile seiner eigenen sozialen Identität und damit des eigenen face mit den übrigen Mitgliedern der Gruppe teilt.

\section{Experten und Laien in medizinischen Hilfsforen}

Einschlägige Studien haben gezeigt, dass die Kommunikation zwischen Experten und Laien wegen unterschiedlicher Wissensbestände der an der Kommunikation Beteiligten generell zu Missverständnissen und Konflikten führen kann; dies gilt in besonderer Weise für die OnlineKommunikation. ${ }^{9}$ Der Wissensvorrat der als (interessierte) Laien bezeichneten Kommunikationspartner kann nicht als gesichert vorausgesetzt werden, weshalb die Sender, die in den untersuchten Foren die Rolle der Experten innehaben, ihre sprachlichen Äußerungen nur unter erschwerten Bedingungen der angenommenen Kompetenz der Mitglieder der discourse community anpassen können. Im Rahmen der Analyse der Experten-Laien-Kommunikation muss zudem diskutiert werden, was grundsätzlich unter einem „Experten“ zu verstehen ist. Da im vorliegenden Beitrag keine face-to-face-Interaktionen, sondern die computervermittelte Kommunikation untersucht wird, ist die Unterscheidung zwischen dem Expertenstatus in der Offline- und in der Online-Identität, der rein diskursiv generiert wird, von Bedeutung:

Expert status represents a measure of authority over a conversation. Expert status refers to a concept very different from that of the status of experts, such as engineers, in society. One might say, using engineering parlance, that the system boundaries for the two concepts are different. The status of experts in society has society as the relevant system. Expert status [...] has the conversation as the relevant system.

(Rifkin/Martin 1997: 31)

Anders als das Kompetenzniveau einer Person in der realen Welt unterliegt der diskursiv ausgehandelte Expertenstatus ständigen Modifikationen. Zudem fallen non-verbale Symbole des Expertenstatus, die in der Offline-Kommunikation Kompetenz signalisieren, in der OnlineKommunikation weg, wodurch den verbalen Strategien eine zentrale Bedeutung zukommt (cf. Park 2008: 2051). In diesem Kontext wird einmal mehr deutlich, in welchem Maße das Vermitteln medizinischer Inhalte - und in diesem Zusammenhang das positive Identitätsmanagement bzw. die Selbstdarstellung als medizinischer Experte - von kommunikativen Prozessen abhängig ist: „Medical knowledge is represented, conveyed and questioned through communicative practices“ (Maci/Sala/Gotti 2015: 9). Auf dem Gegensatz Experte - Laie basierende Machtgefälle werden im virtuellen Raum somit in erster Linie auf kommunikativer Ebene geschaffen.

\section{$5 \quad$ Ergebnisdiskussion}

In den folgenden Abschnitten werden ausgewählte sprachliche Strategien vorgestellt, mittels derer die User in den untersuchten Diskussionsforen zur Medizin ihren eigenen Expertenstatus und denjenigen ihrer Kommunikationspartner aushandeln. In einem ersten thematischen Block stehen Strategien im Mittelpunkt, die darauf abzielen, sich als kompetent in medizini-

\footnotetext{
${ }^{9}$ Cf. die Ergebnisse von Bromme/Jucks/Runde (2005: 90) zur Experten-Laien-Kommunikation im Bereich der Medizin, in deren Kontext das Beziehungsgefüge zwischen medizinischen Experten, Institutionen und Laien grundlegend verändert worden ist: ,the many ways social media and the Web 2.0 have transformed relations between professionals, lay people, and organisations“" (Myers 2015: 54).
}

ISSN 1615-3014 
schen Fragen zu präsentieren. Daran anschließend werden Beispiele diskutiert, in denen sich die User gezielt zurücknehmen und ihren Expertenstatus in Frage stellen.

\subsection{Der Bezug auf eine Autorität}

Durch den Bezug auf eine als kompetent erachtete Autorität sichern die User ihre Aussagen ab, erhöhen ihre Fach- und Urteilskompetenz und erhöhen damit die Hemmschwelle der Mitglieder der discourse community, Kritik an den erteilten Ratschlägen zu üben. Diese als vertrauenswürdige Quelle erachtete Instanz kann eine reale Person sein (der konsultierte Facharzt in Beispiel 1), aber auch eine Studie in Beispiel 2 oder eine Institution (das Deutsche Krebsforschungszentrum) in Beispiel 3. In Beispiel 4 empfiehlt der User seinen Kommunikationspartnern eine Internetquelle, die er als seriös einstuft und der er einen hohen Informationsgehalt zuspricht (,eine Seite wo Du viele Infos dazu findest“); zugleich verweist er auf einen thematisch einschlägigen Vortrag eines namentlich erwähnten Mediziners, der auf der Webseite verlinkt ist. In Beleg 5 untermauert die Tatsache, dass ein praktizierender Arzt die Selbstdiagnose des Users bestätigt hat (,hat jedenfalls sofort meine gestellte Diagnose [...] bestätigt"), dessen medizinische Expertise.

1) Ich habe letzte Woche mit meinem Spezialisten ${ }^{10}$ darüber gesprochen und er hat mir geraten $[\ldots]$.

2) In mehreren, voneinander unabhängigen Studien, die sich mit Darmkrebs beschäftigen, haben Mediziner festgestellt, dass [...].

3) Das deutsche Krebsforschungszentrum hat jüngst darauf verwiesen, dass ein Zusammenhang zwischen der Körpergröße und dem individuellen Risiko, an Krebs zu erkranken, besteht.

4) Hier ist eine Seite wo Du viele Infos dazu findest, es gibt dazu auch eine Studie, da ist auch ein Vortrag von Dr. Bohn zum anschauen.

5) Er schien mir gut zu sein. Hat jedenfalls sofort meine selbst gestellte Diagnose (MCS) bestätigt OHNE dass ich es erwähnt habe

In einigen Fällen diagnostizieren die User auf Basis der Äußerungen der Ratsuchenden (und aufgrund ihrer eigenen Erfahrungen) offenbar den Schweregrad der Erkrankung und weisen sie darauf hin, dass es nicht ausreichend sei, in einem Forum nach Hilfestellung zu suchen. Die Sender machen deutlich, dass ihre eigene Fachkompetenz an Grenzen stößt und legen ihren Kommunikationspartnern nahe, kompetente Personen außerhalb des Forums zu Rate zu ziehen.

6) Sie sollten umgehend einen Fachmann zu Rate ziehen.

7) Was sagt denn Ihr behandelnder Arzt dazu?

In den Belegen 6 und 7 werden die Kommunikationspartner mittels des Modalverbs sollen (,Sie sollten [...] zu Rate ziehen“) bzw. durch eine Frage dazu aufgefordert, einen Spezialisten für Krebserkrankungen bzw. allgemein medizinisches Fachpersonal zu konsultieren. An dieser Stelle fällt auf, dass die reale und die virtuelle Identität in medizinischen Hilfsforen enger miteinander in Bezug zu stehen scheinen als in thematisch anders orientierten Diskussionsforen. Die Tatsache, dass es in medizinischen Belangen um Leben und Tod geht und ein

10 Die für die Beispieldiskussion relevanten Textstellensind fett markiert.

ISSN 1615-3014 
inszenierter und rein fiktiver Expertenstatus ernste Konsequenzen nach sich ziehen kann, führt dazu, dass die User in als problematisch bewerteten Fällen ehrlich auf die Grenzen ihrer Kompetenz verweisen und die Beratung in die reale Welt verlagern, statt auf ihrem diskursiv ausgehandelten Expertenstatus zu bestehen.

Im Kontext der Diskussion, welche Funktionen der Verweis auf externe, als vertrauenswürdig einzustufende Quellen im Korpus haben kann, sei auf die Ergebnisse der Studie von Tereskiewicz (2015) verwiesen, die kommunikative Praxen von Medizinern in englischsprachigen Tweets untersucht hat. Eine zentrale Rolle nimmt dabei, wie in der TwitterKommunikation generell, das Anführen von Links an. In ihrem Korpus beobachtet Tereskiewicz, dass das Posten bestimmter Links mit medizinischer Orientierung dem Bestreben der User entspricht, sich in medizinischen Belangen als kompetent darzustellen und die eigene Glaubwürdigkeit zu erhöhen: „Linking, [...] can be interpreted as a means of establishing and confirming one's professional identity and credibility“ (Tereskiewicz 2015: 79). M. E. kann diese funktionale Ausrichtung des Verlinkens auf der Plattform Twitter mit dem Verweis auf bzw. dem Zitieren kompetenter Quellen in den untersuchten Forenbeiträgen verglichen werden, da es sich in beiden Fällen um eine „quoting practice“ bzw. um den Prozess des „referencing“ (Tereskiewicz 2015: 79) handelt.

\subsection{Der Bezug auf eigene Erfahrungen}

Der Verweis auf eigene Erfahrungen, sei es als mitbetroffene Person, sei es durch eine eigene Erkrankung, verleiht den Usern die Legitimation, den Mitgliedern der discourse community Ratschläge zu erteilen. Zugleich werden die Glaubwürdigkeit und damit das Vertrauen der anderen User in den Sender erhöht. Der Grad an Subjektivität als Nicht-Fachmann wird durch das Herausstellen langjähriger Vertrautheit mit bestimmten Krankheitsbildern reduziert; individuelle Eindrücke erfahren eine „Verwissenschaftlichung“ bzw. Objektivierung, da davon auszugehen ist, dass sich die Betroffenen fundiert über die Erkrankung informiert haben.

8) Bei jedem Patienten spricht die Chemotherapie anders an. Das hängt sicher mit dem Stadium der Erkrankung oder mit der Tatsache zusammen, ob der Körper bereits Metastasen gebildet hat. Ich habe vor 5 Jahren meinen Mann an Bauchspeicheldrüsenkrebs verloren.

9) Vor einem Monat ist meine Mutter an Darmkrebs verstorben.

10) Ich bin jetzt 53 Jahre alt und war vor 10 Jahren ebenfalls an Darmkrebs erkrankt.

11) ich habe seit 30 jahren Diabetis und seit ungefähr 6 Jahren juckende Hände

Während die Userinnen in den Beispielen 8 und 9 ihren Mann bzw. ihre Mutter durch Krebserkrankungen verloren haben, verweisen die Sender in den Belegen 10 und 11 darauf, dass sie seit längerer Zeit an einer bestimmten Krankheit leiden und damit die Erfahrung und die Kompetenz besitzen, ihren Kommunikationspartnern Ratschläge zu erteilen und ihre Expertise mit ihnen zu teilen. 


\subsection{Das Verwenden von Fachterminologie und terminologische Diskussionen}

Durch das Verwenden medizinischer Fachterminologie bzw. metasprachliche Diskussionen bezüglich der Korrektheit verwendeter Fachbegriffe in vorhergegangenen Diskussionsbeiträgen heben die User ihre eigene Expertise hervor. In Beispiel 12 verwendet der User eines Diskussionsforums zu Diabetes den Fachterminus „Polyneuropathie“ nebst der in der medizinischen Fachliteratur üblichen Abkürzung und ergänzt seinen Post durch eine Begriffserklärung in Form einer Definition (,das ist eine nervenstörung, die [...]“).

12) das hört sich bei euch an, als hätten einige von euch eine polyneuropathie (pnp). das ist eine nervenstörung, die meistens bei diabetikern auftritt. bei eurem diabetologen oder noch besser beim neurologen kann das getestet werden.

Auffällig ist in diesem Beispiel das umgangssprachliche Register sowie die explizite Adressatenorientierung der Bedeutungsparaphrase (,,bei eurem diabetologen oder noch besser beim neurologen kann das getestet werden“), die einen Kontrast zum Gebrauch des Fachterminus darstellt. Neben dem Bestreben, die eigene medizinische Expertise herauszustellen, könnte der Gebrauch eines Fachterminus mit anschließender Begriffserklärung in Form einer Äußerung mit geringem Fachlichkeitsgrad somit auch als Strategie der Popularisierung von Wissen und zugleich der Distanzreduktion gewertet werden.

\subsection{Anführen bestätigten Wissens}

In dieser Kategorie sind Belege zusammengefasst, in denen die User als gesichert geltendes medizinisches Fachwissen anführen und auf diese Weise demonstrieren, dass sie über vertieftes Wissen in einem bestimmten Gebiet verfügen.

13) Vereinfacht ausgedrückt, handelt es sich bei Leukämie um eine Krankheit, in deren Verlauf die weißen Blutkörperchen nicht mehr wie gewohnt ihre Aufgaben erfüllen und den eigenen Körper angreifen

14) Um das nochmals richtig zu stellen: Der schützende Effekt des Stillens im Rahmen von Brustkrebserkrankungen ist zwar signifikant, schützt jedoch nicht 100prozentig vor einer Erkrankung

Wie die Belege 13 und 14 demonstrieren, können diese Äußerungen definitorischen Charakter haben, wodurch deutlich gemacht wird, dass das angeführte Wissen nicht hinterfragbar ist. Der Sender weist sich die Rolle des Experten zu, der gegenüber den Empfängern einen Wissensvorsprung besitzt und diese an seinem Wissen teilhaben lässt. Diese inszenierte Divergenz zwischen Experten und Laien manifestiert sich in einigen Fällen mittels metasprachlicher Kommentare wie in Beleg 13, in dem der einleitende Zusatz (vereinfacht ausgedrückt) hervorhebt, dass der Sender über ein profundes Wissen bezüglich der Erkrankung Leukämie verfügt, die folgende Definition aber vereinfacht und an den von ihm als begrenzt bewerteten Wissensstand seiner Kommunikationspartner anpasst. Der Zusatz kann aber zugleich die Funktion eines Abschwächungsmarkers haben, wenn der User deutlich machen möchte, dass die von ihm angeführte Definition keinen Anspruch auf wissenschaftliche Korrektheit erhebt. Auf diese Weise macht er sich weniger angreifbar für Kritik. In Beispiel 14 erfolgt die Korrektur eines vorhergegangenen Diskussionsbeitrags eines anderen Users, einleitend markiert durch um das nochmals richtig zu stellen. Auf der Rezipientenseite kann das Anpassen von 
Äußerungen eines Senders an einen angenommenen Wissensbestand des Kommunikationspartners von diesem sowohl positiv bewertet als auch kritisch betrachtet werden, wenn dieser durch die Popularisierungsstrategie seinen eigenen Expertenstatus hinterfragt fühlt.

\subsection{Kritik an einer Quelle}

Durch die (zumeist explizit geäußerte) Kritik an Quellen, die von anderen Usern als Grundlage ihres medizinischen Wissens zitiert werden, unterstreichen die Sender ihre eigene Urteilskompetenz, da sie Schwächen wie unsauberes methodisches Arbeiten oder das Rezipieren unseriöser Internetseiten offenlegen. In Beispiel 15 distanziert sich der Sender dezidiert von einer Webseite zu medizinischen Fragen, die aus seiner Sicht nicht nur keine brauchbaren Informationen liefert (,keine ernstzunehmenden Informationen zum Thema Diabetes“), sondern - und dies wird als viel schlimmer erachtet - die Gesundheit der Ratsuchenden gefährdet (,die den Krankheitsverlauf verschlimmern können“).

15) Die von Ihnen zitierte Webseite liefert keine ernstzunehmenden Informationen zum Thema Diabetes und gibt den Betroffenen teilweise Ratschläge, die den Krankheitsverlauf verschlimmern können.

16) Der Neurologe hätte die Nervenleitgeschwindigkeit messen müssen.

Kritisieren die User Quellen, die von anderen Mitgliedern der discourse community angeführt werden, wird das face des Kommunikationspartners gefährdet, da dieser in seiner Urteilskompetenz herabgesetzt wird. Wird die Kritik sprachlich zu deutlich geäußert, steht somit das in der Online-Kommunikation wichtige community building, d. h. das Herstellen und Aufrechterhalten sozialer Kohäsion, auf dem Spiel. In Beispiel 16 erhöht der Sender seine eigene Expertise, indem er den aus seiner Sicht fehlerhaften Umgang eines Mediziners mit geschilderten Symptomen kritisiert (,Der Neurologe hätte die Nervenleitgeschwindigkeit messen müssen"); dadurch impliziert er, dass ihm aufgrund seines fundierten Wissens ein solches Fehlverhalten nicht unterlaufen wäre. Da in diesem Beispiel kein Mitglied der Diskursgemeinschaft, sondern ein „Dritter“ bzw. Außenstehender des Forums kritisiert wird, ist das Risiko eines Gesichtsverlusts eher gering. Im Gegenteil: Durch das Solidarisieren mit dem User und dem Ausdruck des Übereinstimmens mit dessen Kritik an einem Arzt leistet der Sender einen Beitrag zum relational work.

\subsection{Ironie und Beleidigungen}

Besonders deutlich manifestiert sich der selbst zugeschriebene Expertenstatus in sprachlichen Äußerungen, die ironisch gefärbt oder gar beleidigend formuliert sind. Durch solche als extrem unhöflich einzustufenden Sprachhandlungen wird nicht nur das Gesicht des Kommunikationspartners verletzt, sondern in gleichem Maße das shared face der discourse community. In der Linguistik herrschte lange Zeit die Auffassung, dass sich die Kommunizierenden in der Online-Kommunikation sehr unhöflich verhalten (cf. Kayany 1998: 1136). Nach Suler (2004: 322-324) sind bestimmte Faktoren der computervermittelten Kommunikation dafür verantwortlich, dass die Kommunikationsteilnehmer eine geringere Hemmschwelle dafür haben, gesichtsbedrohende Sprachhandlungen zu vollziehen. Bedingt durch die Tatsache, dass die Diskussionsteilnehmer unter einem Pseudonym auftreten können und nicht unmittelbar mit den Folgen ihres sprachlichen Handelns konfrontiert werden, zeichnen sich zahlreiche 
Kommunikationsvorgänge in der Online-Kommunikation durch Unhöflichkeit aus (cf. Herring 2007: 16; Maaß 2012). Das Phänomen, in der computervermittelten Kommunikation besonders unhöflich zu agieren, wird in der Forschung als disinhibition effect (,Enthemmungseffekt') bezeichnet.

Die User in den deutschen Diskussionsforen greifen, vermutlich motiviert durch die oben angeführten Risiken der Gesichtsbedrohung, nicht sehr häufig auf ironisch gefärbte oder beleidigende Äußerungen zurück, jedoch sollen exemplarisch einige Belege für dieses kommunikative Verhalten vorgestellt werden. In Beispiel 17 wird der vorhergegangene Beitrag eines Users, der den Gebrauch von Globuli als alternative Behandlungsmethode in der Krebstherapie betrachtet, ironisch kommentiert. Der betroffene User wird, durch das Stellen einer rhetorischen Frage und die Gleichsetzung der Globulitherapie mit Schamanenmedizin, die jeglicher wissenschaftlicher Grundlage entbehre und deren Wirksamkeit nicht nachweisbar sei, öffentlich lächerlich gemacht und es wird ihm jegliche Kompetenz abgesprochen, während sich der Sender als Experte inszeniert. Die laienhafte Äußerung einer Userin hinsichtlich der Therapie einer ernsthaften Erkrankung mit Hilfe von Hausmitteln wird in Beleg 18 mit der ironischen Frage wieder aufgenommen, ob diese jemals versucht habe, ein Getränk zu sich zu nehmen, dessen Temperatur $60^{\circ}$ beträgt. Der Post schließt mit dem belehrenden Kommentar, dass bei dieser Temperatur die Proteine verkleben. Verstärkt wird die Kritik durch die Iteration des Fragezeichens.

17) Glauben Sie wirklich, dass sich Brustkrebs mit Globuli und Schamanenmedizin heilen lässt?

18) Haben Sie je versucht, ein Getränk zu sich zu nehmen, das eine Temperatur von $60^{\circ}$ Celsius hat??? Da verkleben nämlich die Proteine.

Die angeführten Belege demonstrieren den Stellenwert von als unhöflich klassifizierten Sprachhandlungen für den Prozess der Identitätskonstruktion in der Forenkommunikation; sie können als ,a strategic instrument to demonstrate power“ angesehen werden (Kunkel 2014: 406), mit deren Hilfe die Sender ihren (Experten-)Status und ihre soziale Position aushandeln. Der Gebrauch unhöflicher sprachlicher Strategien ist im Spannungsfeld zwischen dem Bedürfnis der User, kommunikative Macht über die anderen Forenmitglieder auszuüben und der Notwendigkeit, soziale Kohäsion zu schaffen, zu verorten.

Auch die folgenden drei Diskussionsbeiträge, in denen mit Nachdruck der Expertenstatus eines anderen Users in Frage gestellt wird, haben das Ziel, die eigene Fach- und Urteilskompetenz bzw. die fachliche Autorität in den Vordergrund zu stellen. Die in den Posts enthaltenen Beleidigungen bergen ein hohes Risiko, den sozialen Zusammenhalt in der Diskursgemeinschaft zu gefährden:

19) Was Sie da von sich geben, hat weder Hand noch Fuß.

20) Ohne weitere Studien zur Entstehung von Diabetes in Ihre Überlegungen mit einzubeziehen, ist Ihr Ratschlag nicht sonderlich hilfreich.

21) Sie wollen hier im Forum den Eindruck vermitteln, dass Sie eine Ahnung davon hätten, wie Tumoren entstehen, ohne jedoch den leisesten Schimmer zu haben, welche Einflussfaktoren bei diesem Prozess eine Rolle spielen. [...] Sie sollten sich erst einmal einen umfassenden Überblick über das Themengebiet verschaffen, bevor Sie anderen Usern falsche Ratschläge erteilen. [...] 
In den Beispielen 19 und 20 hebt der Sender den mangelnden Wahrheitsgehalt (,weder Hand noch Fuß haben“) bzw. die aus seiner Sicht fehlende Relevanz (,Ihr Ratschlag ist nicht sonderlich hilfreich“) eines Diskussionsbeitrags hervor. In Beleg 21 wird die Tatsache kritisiert, dass ein Mitglied der discourse community sich ungerechtfertigter Weise einen Expertenstatus zugewiesen hat, ohne über ausreichendes Fachwissen bezüglich des Entstehens von Krebs zu verfügen (,Sie wollen hier im Forum den Eindruck vermitteln, dass Sie eine Ahnung davon hätten, wie Tumoren entstehen, ohne jedoch den leisesten Schimmer zu haben, welche Einflussfaktoren bei diesem Prozess eine Rolle spielen“). Im Anschluss an diese Kritik folgt der Rat, sich vor dem Erteilen unqualifizierter Ratschläge in das Fachgebiet einzuarbeiten (,Sie sollten sich erst einmal einen umfassenden Überblick über das Themengebiet verschaffen").

\subsection{Das Abschwächen des eigenen Expertenstatus}

Neben dem Herausstellen des eigenen Expertenstatus, häufig bei gleichzeitigem Aberkennen der Expertise anderer User, lassen sich in den untersuchten medizinischen Diskussionsforen Beiträge finden, in denen die User ihren eigenen Expertenstatus abschwächen. In solchen Beiträgen stehen das face work bzw. das Absichern des shared face im Vordergrund. Fachliche Autorität soll nicht zu deutlich in den Vordergrund gestellt werden, um die soziale Kohäsion innerhalb der Diskursgemeinschaft nicht zu gefährden. Zugleich möchten die User vermeiden, aufgrund fehlerhafter medizinischer Ratschläge von ihren Kommunikationspartnern kritisiert zu werden, was sich wiederum negativ auf den Prozess der positiven Identitätskonstruktion auswirken könnte. Hinter den Strategien der Abschwächung können sich somit sowohl eine wahre Unsicherheit auf fachlicher Ebene als auch Bescheidenheit zugunsten des Sicherstellens des relational work verbergen.

22) Ich bin kein Experte auf dem Gebiet der Autoimmunerkrankungen, [...]

23) Wenn ich mich recht erinnere, entsteht Darmkrebs durch die Degeneration von Zellen im Enddarm [...]

24) Rauchen und ein überhöhter Alkoholkonsum spielen vermutlich eine Rolle beim Entstehen von Brustkrebs, aber ich denke, dass ein Zurückführen auf lediglich zwei Faktoren die Krankheit nicht in ihrer Komplexität erklären kann.

25) Sollte ich hier Unsinn von mir gegeben haben, bitte ich um Korrektur!

In Beleg 22 unterstreicht der User, dass er kein Experte auf dem betreffenden Fachgebiet sei („Ich bin kein Experte auf dem Gebiet der Autoimmunerkrankungen“), während die User in den Beispielen 23 (wenn ich mich recht erinnere) und 24 (vermutlich; ich glaube) die eigene Aussage hinsichtlich des Ursprungs einer Erkrankung sprachlich abschwächen (insbesondere durch den Gebrauch des Adverbs vermutlich und des Verbs denken in der 1. Person Singular). Kritik der discourse community wird so im Vorfeld entschärft, da der User sich nicht als unangreifbarer Experte inszeniert, sondern eigene Wissenslücken und Unsicherheiten benennt. Besonders deutlich wird diese Vorsicht beim Äußern medizinischer Ratschläge in Beispiel 25, in dem der User den Kommunikationspartner explizit zum kritischen Hinterfragen einer Aussage auffordert.

Erklären lässt sich das sprachliche Abschwächen des Expertenstatus neben weiter oben angeführten Einflussfaktoren zum einen durch die zu Beginn diskutierten kommunikativen Rah-

ISSN 1615-3014 
menbedingungen der Online-Kommunikation; weiterhin scheinen die thematische Ausrichtung medizinischer Foren und die Interdependenz von realer und virtueller Sphäre eine Rolle zu spielen. Da den Usern bewusst ist, dass ein falscher oder missverständlicher Rat gravierende Folgen haben kann, wird in einzelnen Fällen zugunsten ehrlicher Selbstzweifel oder des Hinweises auf die eingeschränkte Gültigkeit von Aussagen auf die diskursive Inszenierung von Expertise verzichtet.

26) Jedoch halte ich es für wichtig, zumindest war es das für mich, zu jedem einzelnen Arzt gegangen zu sein.

27) Leider kann ich nach so langer Zeit auch nicht viel dazu beitragen. Ich war auch bei 100 Ärzten, mit Gehirnwasser ziehen etc. ... Alles ohne Befund. Für einen Austausch bin ich aber jederzeit gern offen.

Vorsicht beim Äußern medizinischer Ratschläge manifestiert sich weiterhin sprachlich in den Belegen 26 und 27. In Beispiel 26 subjektiviert der User einen vom ihm erteilten Ratschlag stark (halte ich es für wichtig; die gleiche Funktion hat der Einschub jedenfalls war es das für mich), wodurch dieser relativiert wird. Ein Anspruch auf Allgemeingültigkeit durch den User wird nicht erhoben. Diskussionsbereitschaft mit den anderen Mitgliedern wird in Beleg 27 signalisiert.

\section{Schluss}

Die Auswertung eines Korpus deutschsprachiger Diskussionsforen aus dem Bereich der Medizin hinsichtlich des sprachlich-diskursiven Aushandelns des Expertenstatus der Kommunikationsteilnehmer hat ergeben, dass zwei diametral entgegengesetzte Strategien verfolgt werden. In den meisten Fällen sind die Diskussionsteilnehmer bestrebt, ihren eigenen Expertenstatus sprachlich hervorzuheben und somit ihre Online-Identität möglichst positiv zu beeinflussen. Dabei erfolgen nicht nur positiv wertende Stellungnahmen hinsichtlich der eigenen Expertise, sondern auch kritische Evaluierungen der Fachkompetenz der Kommunikationspartner. Seltener werden in den analysierten Diskussionsforen die eigene Kompetenz in Frage gestellt, Wissenslücken zugegeben und die eigene fachliche Autorität abgeschwächt. Jedoch lässt sich dieses für die Online-Kommunikation eher untypische Verhalten durch die thematische Besonderheit medizinischer Diskussionsforen begründen: Ein falscher Ratschlag kann gravierende Folgen in der Offline-Realität haben, woraus eine tendenziell vorsichtige Kommunikation und im Zweifelsfall das Zurückstellen des virtuellen Aushandelns des Expertenstatus resultieren. Für den untersuchten Forentyp sind die Online- und die Offline-Realität vergleichsweise eng miteinander verknüpft. ${ }^{11}$

Die Analyse hat gezeigt, dass die Forenteilnehmer unterschiedliche sprachliche Strategien anwenden, um ihren Expertenstatus in der Diskussion zu unterstreichen. Dazu gehört in vielen Fällen der Bezug auf eine Autorität, die sich in Form einer Fachpublikation, einer Institution oder einer als kompetent erachteten Person manifestieren kann. Durch diesen Bezug werden einerseits die eigenen Aussagen abgesichert, legitimiert und weniger angreifbar gegen die

\footnotetext{
${ }^{11}$ Cf. die Studie von Kunkel (2014) zum Aushandeln des Expertenstatus in französischen Diskussionsforen zu sprachnormativen Fragen: Kunkel stellt heraus, dass die User in diesem Diskursbereich hingegen ausnahmslos bestrebt sind, ihren eigenen Expertenstatus mittels sprachlicher Strategien zu festigen und zu diesem Zweck häufig auf unhöfliche Sprachhandlungen zurückgreifen.
}

ISSN 1615-3014 
Kritik der Diskursgemeinschaft gemacht. Zudem heben die Sender ihre eigene Fachkompetenz bzw. Belesenheit hervor, insofern es sich um schriftliche Quellen wie z. B. medizinische Studien oder Fachaufsätze handelt. Der Verweis auf die Kenntnis einschlägiger Institutionen des Medizinbetriebs demonstriert weiterhin eine gute Vertrautheit mit der Disziplin. Andererseits wird durch die Referenz auf medizinisches Fachpersonal als kompetenter Instanz die Begrenztheit der eigenen Expertise in den Vordergrund gestellt. Eine weitere zentrale Strategie im Kontext der positiven Identitätskonstruktion besteht in der Narration einer eigenen Krankheitsgeschichte oder im Verweis auf Erfahrungen mit bestimmten Krankheiten, die dem Sender nahe stehende Personen durchgemacht haben. Diese Berichte bzw. Verweise erhöhen die Glaubwürdigkeit des Senders und verleihen seinen Ratschlägen Legitimität. ${ }^{12}$ Das Verwenden medizinischer Fachterminologie und der metasprachliche Verweis auf einen korrekten terminologischen Gebrauch werden ebenfalls mit dem Ziel eingesetzt, die eigene Kompetenz $\mathrm{zu}$ unterstreichen. Weiterhin führen die Sender allgemein anerkanntes medizinisches Wissen an, häufig in Form von Definitionen, um herauszustellen, dass dieses Wissen nicht hinterfragbar ist. Durch diese Strategie wird die Diskrepanz hinsichtlich des Wissensstands zwischen „Experten“ und „Laien“ sprachlich akzentuiert. In den Fällen, in denen die Sender Kritik an Quellen üben, die von anderen Mitgliedern der discourse community herangezogen werden, wird die eigene Urteilskompetenz in das Zentrum gerückt. Diese Selbstzuschreibung des Expertenstatus birgt im Falle kritischer Kommentare zu von Kommunikationspartnern angeführten Quellen allerdings das Risiko des Gesichtsverlusts und des Gefährdens der sozialen Kohäsion zwischen den Forenteilnehmern. Aufschlussreich sind weiterhin die Belege des Korpus, in denen die Sender durch ironische Kommentare oder Beleidigungen die Expertise der Kommunikationspartner in Frage stellen. Solche extrem unhöflichen Sprachhandlungen gefährden das shared face der Diskussionsteilnehmer, sind aber zugleich ein wichtiges Instrument der Identitätsbildung in der Online-Kommunikation (cf. Kunkel 2014: 414).

Relativ selten treten in den Foren Hedging-Strategien auf, die dem Abschwächen des eigenen Expertenstatus dienen. Die Diskussionsteilnehmer setzen diese Strategien mit dem Ziel ein, face work zu betreiben und sich nicht über die Expertise ihrer Kommunikationspartner zu erheben. Zugleich widerspiegeln Hedging-Strategien aber auch die Vorsicht der Sender, medizinische Ratschläge zu erteilen, die ggf. schwerwiegende Folgen in der Realität haben können.

Im Rahmen einer weiteren Studie sollen die Ergebnisse der Analyse deutschsprachiger Diskussionsforen zur Medizin mit thematisch anders orientierten Foren verglichen werden, um eventuell bestehende forenspezifische sprachliche Strategien zum Aushandeln des Expertenstatus zu identifizieren.

\section{Korpus}

www.gesundheit.com. https://www.gesundheit.de/ [10.12.2015] www.gesundheit.de. https://www.gesundheit.com/ [10.12.2015] www.onmeda.de. www.onmeda.de [10.12.2015]

\footnotetext{
12 Das Herausstellen eigener Erfahrungen ist ein weit verbreitetes Muster in der Forenkommunikation, das zu kontroversen Diskussionen zwischen den Kommunikationspartnern führen kann. Personen, die keine Erfahrungen mit bestimmten Sachverhalten haben, zu denen sie sich äußern, werden häufig kritisiert.
} 


\section{Literatur}

Baumann, Eva/Hurrelmann, Klaus (2014): „Gesundheitskommunikation: Eine Einführung“. In: Baumann, Eva/Hurrelmann, Klaus (eds.): Handbuch Gesundheitskommunikation. Bern, Huber: 8-17.

BITKOM (2011): „Das Internet wird zum Gesundheitsratgeber“. www.bitkom.org/de/markt _statistik/64026_69111.aspx [15.10.2015].

Bromme, Rainer/Jucks, Regina/Runde, Anne (2005): "Barriers and biases in computermediated-expert-layperson-communication”. In: Bromme, Rainer/Hesse, Friedrich W./Spada, Hans (eds.): Barriers and Biases in Computer-Mediated Knowledge Communication. And How They May Be Overcome. Dordrecht, Kluwer: 89-118.

Ehrhardt, Claus (2011): „Höflichkeitsbegriffe - am Beispiel von Höflichkeit und Höflichkeitsbewusstsein in Internetforen“. In: Ehrhardt, Claus/Neuland, Eva/Yamashita, Hitoshi (eds.): Sprachliche Höflichkeit zwischen Etikette und kommunikativer Kompetenz. Frankfurt a. M., Lang: 27-44.

Ehrhardt, Claus (2014): "Politeness and Face Work in German Forum Communication". In: Bedijs, Kristina/Held, Gudrun/Maßß, Christiane (eds.): Face Work and Social Media. Münster, LIT: 83-107.

Herring, Susan C. (2007): "A Faceted Classification Scheme for Computer-Mediated Discourse”.Language@Internet 4: article 1.www.languageatinternet.org/articles/2007/761 [15.10.2015].

Hurrelmann, Klaus/Leppin, Anja (2001): „Moderne Gesundheitskommunikation - eine Einführung“. In: Hurrelmann, Klaus/Leppin, Anja (eds.): Moderne Gesundheitskommunikation. Vom Aufklärungsgespräch zur E-Health. Bern, Huber: 9-21.

Kaltenborn, Karl-Franz (2001): „Medizin- und gesundheitlicher Wissenstransfer durch Medien“. In: Hurrelmann, Klaus/Leppin, Anja (eds.): Moderne Gesundheitskommunikation. Vom Aufklärungsgespräch zur E-Health. Bern, Huber: 36-69.

Kayany, Joseph M. (1998): "Contexts of uninhibited online behavior: Flaming in social newsgroups on usenet". Journal of the American Society for Information Science and Technology 49/12: 1135-1141.

Kreps, Gary L./Bonaguro, Ellen W./Query, Jim L. (1998): “The history and development of the field of health communication". In: Jackson, Lorraine D./Duffy, Berna K. (eds.): Health communication research. Westport/CT, Greenwood: 1-16.

Kristiansen, Silje/Bonfadelli, Heinz (2013): „E-Health: Gesundheit im Internet“. In: Rossmann, Constanze/Hastall, Matthias (eds.): Medien und Gesundheitskommunikation. Befunde, Entwicklungen, Herausforderungen. Baden-Baden, Nomos: 237-255.

Kunkel, Melanie (2014): "Impoliteness in the negotiation of expert status". In: Bedijs, Kristina/Held, Gudrun/Maßß, Christiane (eds.): Face Work and Social Media. Münster, LIT: 403-421.

Maaß, Christiane (2012): „Der anwesende Dritte im Internetforum“. In: Bedijs, Kristina/Heyder, Karoline Henriette (eds.): Sprache und Personen im Web 2.0. Münster, LIT: 73-93.

Maci, Stefania M./Sala, Michele/Gotti, Maurizio (2015): “Introduction”. In: Gotti, Maurizio/Maci, Stefania/Sala, Michele (eds.): Insights into Medical Communication. Bern etc., Lang: 9-24. 
Marwick, Alice/Boyd, Danah (2010): "I Tweet Honestly, I Tweet Passionately: Twitter Users, Context Collapse, and the Imagined Internet”. New Media and Society 13/1: 114-133. doi: $10.1177 / 1461444810365313$.

Myers, Greg (2015): “Social Media and professional Practice in Medical Twitter”. In: Gotti, Maurizio/Maci, Stefania/Sala, Michele (eds.): Insights into Medical Communication. Bern etc., Lang: 51-69.

Park, Jung-ran (2008): "Linguistic politeness and face-work in computer-mediated communication. Part 1: A theoretical framework". Journal of the American Society for Information Science and Technology 59/13: 2051-2059.

Rehm, Georg (2005): Hypertextsorten. Definition - Struktur - Klassifikation. Unveröffentlichte Dissertation, Justus-Liebig-Universität. geb.uni-giessen.de/geb/volltexte/2006/2688/ [01.10.2017].

Rifkin, William. D./Martin, Brian (1997): "Negotiating expert status: Who gets taken seriously”. IEEE Technology and Society Magazine 16/1: 30-39.

Schröder, Tilman (2013): Marketingstrategien auf Unternehmenswebsites im internationalen Vergleich. Eine hypertextlinguistische und kulturkontrastive Analyse kommerzieller Websites aus Deutschland, Frankreich, Spanien, Großbritannien und den USA. Tübingen: Narr.

Schröder, Tilman (i. Ersch.): „Ernährungsratgeber in deutschen und französischen Gesundheitsportalen. Eine digitale Textsorte im Spannungsfeld von Fachinformation und Publikumsorientierung“". In: Döring, Martin/Rentel, Nadine (eds.): Medizinische Linguistik des Französischen. Fachsprachliche und medienlinguistische Perspektiven. Bonn: Romanistischer Verlag.

Suler, John (2004): “The Online Disinhibition Effect". Cyberpsychology \& Behavior 7/3: 321-326.

Tajfel, Henri (1974): "Social identity and intergroup behaviour". Social Science Information 13/2: 65-93.

Tereszkiewicz, Anna (2015): "Medical Doctors on Twitter: How and Why MDs Tweet". In: Gotti, Maurizio/Maci, Stefania/Sala, Michele (eds.): Insights into Medical Communication. Bern etc., Lang: 71-92.

Thaler, Verena (2012): Sprachliche Höflichkeit in computervermittelter Kommunikation. Tübingen: Narr.

Tienken, Susanne (2013): „Sharing. Zum Teilen von Erzählungen in Onlineforen”. In: Álvarez López, Laura/Seiler Brylla, Charlotta/Shaw, Philip (eds.): Computer-mediated discourse across languages. Stockholm, Acta Universitatis Stockholmiensis: 17-43.

Warm, Johanna (2015): „Beziehungs- und Identitätsarbeit auf der Social Network Site Facebook“. In: Polzin-Haumann, Claudia/Gil, Alberto (eds.): Angewandte Romanistische Linguistik. Kommunikations- und Diskursformen im 21. Jahrhundert. St. Ingbert, Röhrig Universitätsverlag: $285-301$.

Zappavigna, Michele (2011): “Ambient Affiliation: A Linguistic Perspective on Twitter”. New Media \& Society 13/5: 788-806. doi: 10.1177/1461444810385097. 